\title{
Selection of Parameters in Active Contours for the Phenotypic Analysis of Plants
}

\author{
$\underline{\text { Josh Chopin }}^{\text {a,b }}$, Stanley J. Miklavcic ${ }^{\text {a,b }}$, Hamid Laga ${ }^{\text {a,b }}$ \\ ${ }^{a}$ Phenomics and Bioinformatics Research Centre, \\ School of Information Technology and Mathematical sciences, University of South Australia \\ Mawson Lakes, SA 5095, Australia \\ ${ }^{b}$ Australian Centre for Plant Functional Genomics \\ Plant Genomics Centre, Hartley Grove, Urrbrae \\ PMB 1 Glen Osmond, SA 5064, Australia \\ Email: joshua.chopin@unisa.edu.au
}

\begin{abstract}
Modern genomics experiments are often conducted in controlled environments on hundreds of plants at a time. As such, manual and destructive phenotyping techniques are no longer suitable. Recently there has been a trend toward automated high-throughput phenotyping facilities, in an attempt to relieve the phenotyping bottleneck. A majority of these facilities focus on the use of cameras for non-destructive recording of plant data. Hence, the new challenge lies in accurately segmenting the plants from $2 \mathrm{D}$ images in an automated manner and then analysing structural and statistical information about them.
\end{abstract}

One such technique for image segmentation is known as the active contour model. Active contour models have seen widespread success throughout a number of applied fields due to their versatility and semiautomated nature. However, a high majority of these models rely on arbitrary parameters that are required to be selected manually. Furthermore, small variations in these parameters can produce substantial variations in the method's overall accuracy. This makes them unsuitable for use by non-experts and also for the analysis of a series of images that can change significantly over time. For example an image sequence for the growth of a plant.

In this paper we attempt to establish relationships between the parameter values of active contour models and the geometry of the objects/shapes that they are segmenting. Our goal is for users to be able to utilise some basic a-priori knowledge about the geometry of the object in order to automatically select a range of suitable parameter values. We analyse the accuracy of active contour models over multiple series of shapes that exhibit some pattern, such as decreasing number of sides or increasing concavity. We present a novel normalization technique so that the parameter values are of a similar scale. We also carefully design an experimental setup that ensures no bias between different shapes or parameter values.

We show that over a series of shapes the range of parameters that provide convergence do follow a trend. We also show that not all contours that converge to the objects boundary do so in a stable manner, with a substantial amount oscillating continuously. However more information, such as more shapes and more parameter values, is required to draw meaningful and quantitative conclusions from such an analysis. Future work includes incorporating more of this information along with the application to more active contour models. Another exciting future direction is the use of $2 \mathrm{D}$ shape diagrams to quantify relationships between shapes, parameter values and levels of accuracy.

Keywords: Active contours, parameter selection 


\section{INTRODUCTION}

The seminal work of Kass \& Witkin (1988) introduced a revolutionary curve evolution technique, known as active contour model, for the image segmentation problem. It provided a means of non-interactive segmentation using both image information and mathematical formulations of 2D curve propagation. Since their inception, active contour models have been studied extensively and resultantly the models' capabilities have evolved substantially. The first active contour model, proposed by Kass and Witkin (1988) and known colloquially as 'snakes', was a 2D parametric curve defined in the image domain, ideally attracted to object boundaries. Since then, active contour models have found more recent success due to being defined by implicit functions that are capable of changes in topology, robustness toward noise and attraction to image gradients, intensities and features of interest. However, despite numerous advances in the field, one characteristic of active contours that has gone unchanged since their inception in 1988 is the requirement of manually choosing values for a number of ambiguous parameters. In practice, it appears that only slight variations in object shape or image type can demand vast changes in the selection of these parameters.

The versatility of these models makes them an appealing choice for a wide array of image types and segmentation scenarios. As such, active contour models have been used in various fields, such as robotics (Farag et al. 2007), medical imaging (Stijnen et al. (2009)), satellite imaging (Argialas and Karantzalos (2009).), computer graphics (Museth et al. 2004), optimal control (Ring and Hintermuller (2004)) and plant phenotyping (Frick et al. 2011). In most of these applications, the parameters of the active contour models should be tuned manually. In high-throughput scenarios, however, the manual tuning of parameters severely limits the usage of active contour models. In particular, in plant genomics experiments where accurate plant phenotyping is used to measure experiment success, numerous features must be extracted from hundreds of images on a daily basis, in order to enable plant growth modelling over time or biomass estimation. Active contour models exhibit many characteristics that are appealing to plant phenotypers, but the arduous task of manual parameter selection for different plants at different stages of growth and for various types of backgrounds and lighting conditions, ultimately make them currently unsuitable for the application.

Recently there has been a steady growth of literature either analyzing or attempting to solve the parameter selection problem for active contours. Cai et al. $(2006,2009)$ use a number of training images with known ground truth to implement a genetic search algorithm for finding optimal parameters. Support Vector Machines (SVM) were then used to define a relationship between image characteristics and active contour parameters that could be used to select parameters for future images. While this work was applied to the segmentation of roads from satellite images a similar approach was taken in the work of Iakovidis $e t$ al. (2007) for segmenting thyroid ultrasound images and Kussener (2011) who also employed parallel computing. While these machine learning type techniques often yield good results they require a large amount of data for the learning stage, which isn't always available.

Another approach being investigated more recently is to use logical image information to dynamically update the parameters as the method progresses. Allili et al. (2008) propose a Bayesian model where the parameters are treated as hyper-parameters to be estimated. Mylona et al. (2012) and Zhu et al. (2011) also propose methods to dynamically update parameter values. However, their techniques update parameters spatially, selecting different values for different regions of the evolving curve.

In the majority of traditional literature introducing new active contour models the authors provide a set of parameters that have been chosen through experimentation to align well with their method and the images used. Tian et al. (2013) take the parameter selection one step further by performing a short sensitivity analysis on the parameters required for their novel method. The authors create a table displaying the different parameter values used to gain optimal segmentation for a number of different image and object types. Furthermore, they produce plots showing the variation in error as the parameters are changed and remark upon various relationships that seem to occur between parameter values and image types. They conclude that three of their parameters are relatively stable but the remaining four are quite sensitive. Furthermore, the authors provide a threshold for these parameter values to avoid local minima and unreasonable accuracy.

In this exploratory work we take the analysis of Tian et. al (2013) one step further and attempt to establish universal relationships between these parameters and the objects/images they are segmenting. We will focus on the snakes of Kass and Witkin (1988), as they are the foundation for modern day active contour models and present a simply defined geometrical formulation. We postulate that successful combinations of object geometry and parameter values will also apply to more current active contour models and prescribe this as one direction for future work. Through experimentation, we analyse the role that each of the parameters play in the snake's evolution and hence which parameters are suitable for the geometric properties of different objects. We also analyse the problem of fast convergence, while outlining the problem of image noise for 
future work. Ultimately, using all of this information, we plan to develop an algorithm for automated parameter selection requiring only the most basic of a-priori knowledge.

This paper is organised as follows: In Section 2 we provide an overview of the formulation of snakes and the role that each parameter plays in the curves evolution. Section 3 details the methodology behind our parameter analysis experiments. The results of these experiments are presented and discussed in Section 4. In Section 5 we provide the conclusions of our work and outline which facets of parameter analysis are still required and provide strategies for conducting this analysis in the near future.

\section{PARAMETERS FOR ACTIVE CONTOUR MODELS}

Active contours are 2D curves that evolve/deform over time, such that a predefined energy is minimized. This energy is the summation of different forces, which can be broadly split into two categories; internal forces and external forces. In this section we provide formal definitions for both the $2 \mathrm{D}$ curve itself and the forces that drive its evolution. We will also discuss the geometric significance of these forces.

Let $C(q)=(x(q), y(q))$ be a parameterized curve such that $C:[0,1] \rightarrow \mathbb{R}^{2}, q \mapsto(x(q), y(q)), C$ is piecewise continuous and each continuous piece is at least second order differentiable. We define the energy of the curve $C$ as:

$$
E_{\text {snake }}(C(q))=\int_{0}^{1}\left[E_{\text {int }}(C(q))+E_{\text {image }}(C(q))\right] d q
$$

where $E_{\text {int }}$ and $E_{\text {image }}$ are respectively the internal and external energies. The internal energy is derived from intrinsic properties of the curve itself, such as length and curvature. Since $C$ is parameterized on the domain $[0,1]$, the length of $C$ is defined simply as $L=\int_{0}^{1}\left|C^{\prime}(q)\right| d q$. Also, since $C^{\prime}(q)$ gives the tangent to the curve at point $C(q)$ and curvature is defined as the derivative of the tangent, the curvature at point $C(q)$ is given by $\kappa(q)=\left|C^{\prime \prime}(q)\right|$. The external energy is designed to attract the snake to the desired features of the object using properties of the image itself. Kass and Witkin claimed that the edges of the object provide a good approximation to its boundary. Hence, they decided that the active contour's external energy should attract it to edges in the image. Using only image information, 'edges' correspond to regions of high gradient, therefore we can formulate the external energy as $E_{\text {image }}(C(q))=\nabla I_{G}(C(q)) d q$, where $\nabla I_{G}(C(q))$ is the gradient of the image $I$, which has been smoothed with a Gaussian $G$, at point $C(q)$. In summary, snakes are curves in $2 \mathrm{D}$ space that attempt to iteratively reduce their size while locking onto object boundaries and stopping there. Using these definitions of internal and external energies, the Kass and Witkin snakes formulation is defined as follows:

$$
E(C)=\alpha \int_{0}^{1}\left|C^{\prime}(q)\right|^{2} d q+\beta \int_{0}^{1}\left|C^{\prime \prime}(q)\right|^{2} d q-\lambda \int_{0}^{1}\left|\nabla I_{G}(C(q))\right|^{2} d q
$$

The first term, referred to as the $\alpha$ term, minimizes the contours length. The $\beta$ term constrains the contour to smoothness by minimizing its curvature. The $\lambda$ term minimizes the contour's distance from image edges.

The importance of parameter selection in the snakes formulation is immediately clear when we consider the following example scenario. Figure 1a and Figure $1 \mathrm{~b}$ show a grayscale image of an infant wheat plant, with a converged snake shown in red. We initialized a contour $C_{0}$ around the outside of the plant in each image and set $\alpha=0.05, \beta=0, \lambda=8$ and $\alpha=0.001, \beta=0, \lambda=100$, respectively. As it approaches the leaf tip at the top of the image, the evolution of the contour will be sped up by the $\alpha$ and $\beta$ terms attempting to minimize its length and curvature, respectively. At the same time, the $\lambda$ term is attempting to draw the contour as close as possible to the objects edges. As a result the method fails to accurately capture the leaf tip in Figure 1a, whereas in Figure $1 \mathrm{~b}$ the larger $\lambda$ value keeps the contour close to the object boundary. Immediately we can see a contradiction between the internal and external forces of the snake formulation. In these scenarios, the weighting that the user specifies for each force through the $\alpha, \beta$ and $\lambda$ parameters can have a significant impact on the contours accuracy at convergence.

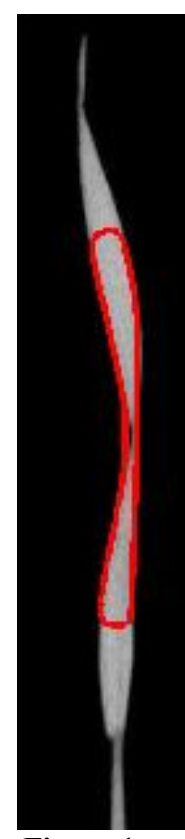

Figure 1a:

Contour with

$\alpha=0.05$,

$\boldsymbol{\beta}=\mathbf{0}, \boldsymbol{\lambda}=8$

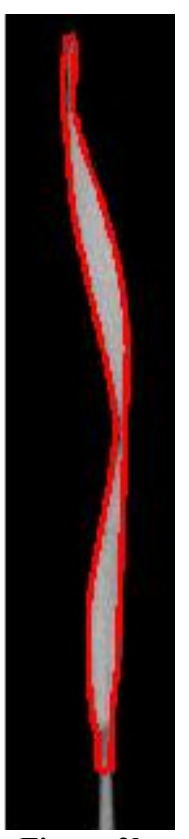

Figure 2b: Contour with $\alpha=\mathbf{0 . 0 0 1}$ $\boldsymbol{\beta}=\mathbf{0}$, $\lambda=100$ 
The fundamental limitation present in applying snakes to automated phenotypic analysis is accuracy. While the method is, in theory, automated fixing a constant set of parameters will not provide any reasonable level of accuracy for plants at different stages of growth, of different species, and imaged in different backgrounds and under various lighting conditions. This is in particular a large limitation for plant phenotypers as a number of phenomena throughout the plants growth require accurate measurement of very fine details. For example, plant phenotypers are interested in knowing when a plant begins to senesce, which occurs primarily at the very tip of plant leaves, or when the plant first develops a flag leaf.

To the best of our knowledge, the parameter selection problem has not been investigated by the current literature. A large majority of applied work provides results sections containing a table of suitable parameter values for their specific purposes. In this paper, rather than providing a list of parameters suitable for our specific application, we will analyse why that list provides a reasonable level of accuracy and determine relationships between parameter values, object geometry, convergence rates and accuracy.

\section{METHODOLOGY}

\section{Shape Selection}

Figure 2 shows the range of shapes that we have studied. The rows represent series of shapes with a pattern occurring, such as increasing number of edges or decreasing convexity. Analysing properties of the active contours over a set of shapes that exhibit a specific pattern provides us insight into how the range of successful parameters changes for different changes in geometry. This simple database was used rather than popular shape databases, such as the MPEG 7 shape database, as it is created specifically for analyzing certain geometric properties and evolution of shapes.

\section{Experimental Setup}

All images were 400x400 pixels in dimension. To ensure that there was no bias toward shape geometry, all shapes were created with identical areas. They were placed in the centre of the image such that the midpoint of the image's $x$ values i.e. $x=200$, corresponds to the midpoint between the shape's minimum and maximum $x$ values and similarly for $y$. Furthermore, the snakes were all initialized using the same square curve, so that for each experiment the proportion between initial contour area and object area remained constant. This experimental setup ensured that, for perfect segmentation, each contour would have to travel over the same number of pixels for each shape. Hence, our experiments do not introduce any bias on convergence rates between shapes.

Each contour was evolved for exactly 6000 iterations. Although various criteria can be used to check the convergence of the algorithm, they introduce yet another parameter to the process. Rather than implementing such a technique we chose to let the snakes evolve for a large, constant number of iterations. Also, even when convergence criteria is specified, there is no guarantee that the contour will converge, so there is usually a limit set on the maximum number of iterations. In our experiments we record statistics about the contour at every iteration, so it was possible to define convergence criteria retrospectively and avoid the risk of stopping the method too early.

\section{Normalization}

In a regular setting, the value chosen for $\lambda$ is often between 100 and 10,000 times larger than that of $\alpha$. In a standard binary or rescaled gray level image, where pixel values are in the range of $[0,1]$, the greatest value that the image gradient can take at any point is one. Even if the grayscale values remain between 0 and 255, the greatest value that the gradient can take is 255 . Alternatively, the length of the contour can become arbitrarily large, depending on image size. Hence, the value of $\lambda$ is often required to be much larger than that of $\alpha$.

Rescaling the intensity values of the image, to increase the magnitude of the gradient, will not work in a general sense as they would have to be rescaled to suit the length of the contour, which is variable depending on image size. Rather, since we know that gradient values will always lie between 0 and 1 , we decided to rescale the contour itself, so that the force provided from the contour length will be of a similar magnitude. To do so, we divide each coordinate point by the size of the image, to acquire a domain for $x$ and a range for $y$ that are both between 0 and 1 . Granted, the length of the contour will not always be between 0 and 1 , since the contour can enlarge as well as shrink, but unlike prior to rescaling the two forces will now be of comparable magnitudes. 


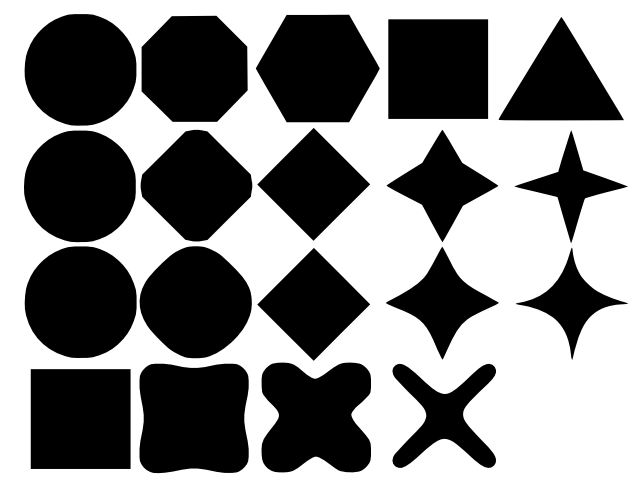

Figure 3 Diagram of all shapes used for parameter analysis. Series of shapes are given in rows.

\section{Accuracy}

To calculate the accuracy of a converged contour we use the measure defined in (Berg, 1998). If we think of the interior of the converged contour as a set of points, $S_{1}$, and the interior of the ground truth shape to also be a set of points, $S_{2}$, then the error is defined as:

$$
E\left(S_{1}, S_{2}\right)=\left(S_{1} \cup S_{2}\right) \cap\left(S_{1} \cap S_{2}\right)^{c}
$$

This is the set symmetric difference operator, which provides us the sum of the number of pixels inside the contour, which are not inside the shape and the number of pixels inside the shape but not inside the contour.

\section{RESULTS AND DISCUSSION}

Although this work is only at a preliminary stage a number of results have already been gathered. Figure 3 shows a 2D plot of $25 \alpha$ values and $25 \lambda$ values and classifies each pair into one of three categories. For each of the 625 combinations of $\alpha$ and $\lambda$ tested we determine whether; A - The contour jumped over the boundary of the object and converged to a point, B - The contour converged to somewhere on the objects boundary or $\mathrm{C}$ - The contour converged to somewhere on the objects boundary but continued to oscillate around that solution. Using this information we have defined these three regions in Figure 3, with the blue region illustrating parameter values that fall into category A, the brown region for category $\mathrm{B}$ and the green region for category $\mathrm{C}$.

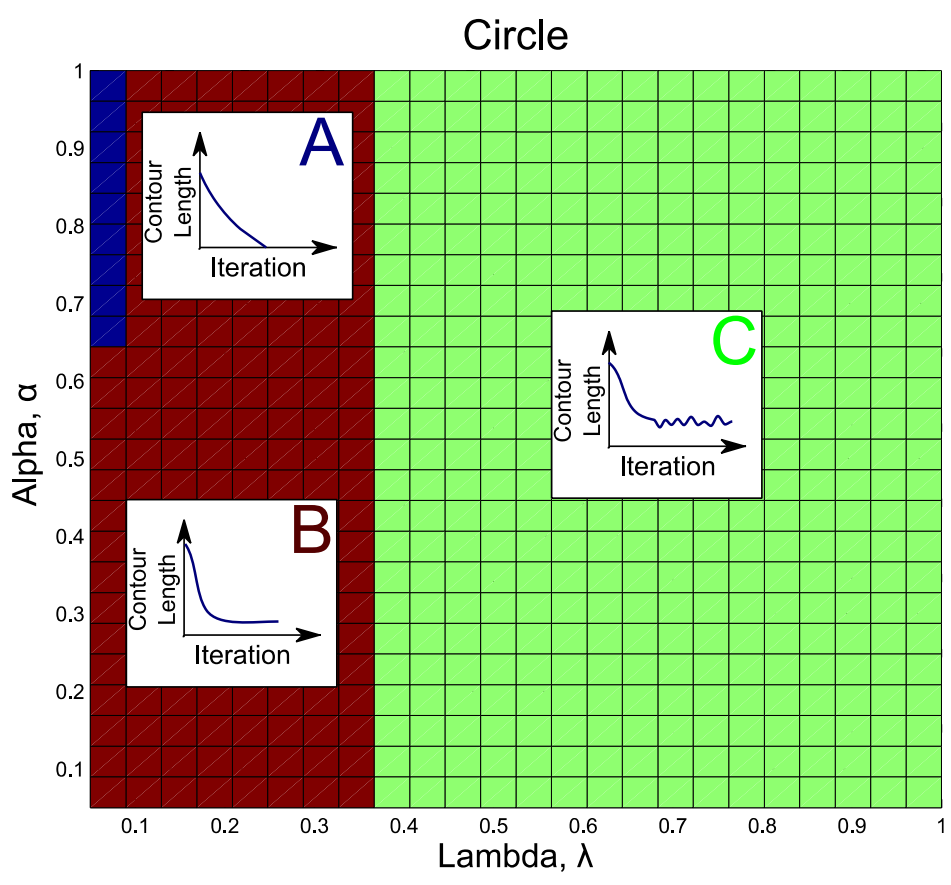

Figure 4 Plot representing which values of $\boldsymbol{\alpha}$ and $\boldsymbol{\lambda}$ result in which type of convergence for the case of a circular image object. Region A - Contour converging to a point, Region B - Stable convergence and Region C - Oscillating convergence. 
The classification defined in Figure 3 provides no information about accuracy. However, it is important to note that solutions from both region $\mathrm{A}$ and region $\mathrm{B}$ can be of a reasonable accuracy, whereas the solutions in region A will never be desirable. As such, we would ideally like to be able to predict where this region lies, so that users can select parameter values that avoid it. As we hypothesised, the location and area of this region varies between different geometric shapes. Furthermore, these variations follow a pattern through a series of shapes. Figure 4 is a contour plot, showing the boundary between the desirable and undesirable regions for the series of shapes from the first row of Figure 2. For example, the red contour in Figure 4 corresponds to region $\mathrm{A}$ in Figure 3, which was for the circle image. The next object in the series is the octagon, illustrated with the blue contour. We can see that region A, although similar in shape now spans a few more $\lambda$ values and a substantial amount of $\alpha$ values. This trend continues through the rest of the shape series.

\section{CONCLUSIONS AND FUTURE WORK}

Active contour models are undoubtedly a useful tool for image segmentation throughout a variety of fields. However the parameter selection problem limits their usefulness in high throughput scenarios and their usage by non-experts. In this work we outlined the roles that these parameters plays and provided reasoning behind their sensitivity. We have introduced a novel normalization technique to enable two of the methods main parameters to be on a similar scale and also analysed the method over a reasonable number of combinations of these parameters. We show that through different series of shapes the parameters that provide the method with stability exhibit somewhat predictable trends. However more information, such as more shapes and more parameter values, is required to do so accurately.

In this exploratory work we have analysed the accuracy and convergence of snakes with two different parameter values. The two parameters that we chose to investigate are important as they provide weights to the two guiding forces of the contour. However, they are not the only parameters present in the model. In future work we aim to extend this analysis to the $\beta$ and $\sigma$ parameters, where $\sigma$ is the standard deviation of the Gaussian that smoothes $I$.

Furthermore, snakes are only one particular active contour model and many other, more recent, models with their own parameters remain for future work. We postulate that models with a similar energy formulation will exhibit similar ranges of successful parameters.

Currently the selection of shapes and connection between shape and parameter values is somewhat qualitative. To provide a more mathematical interpretation of this connection we plan to employ shape diagrams (Rivollier, 2011) that map each shape to a point in 2D Euclidean space. Ideally, we will also be able to map regions of this $2 \mathrm{D}$ space to ranges of acceptable parameters for various active contour models. Furthermore, as image noise will determine the usefulness of local image information, we will include 'amount of noise' as a piece of basic a-priori knowledge to help select correct parameter values.

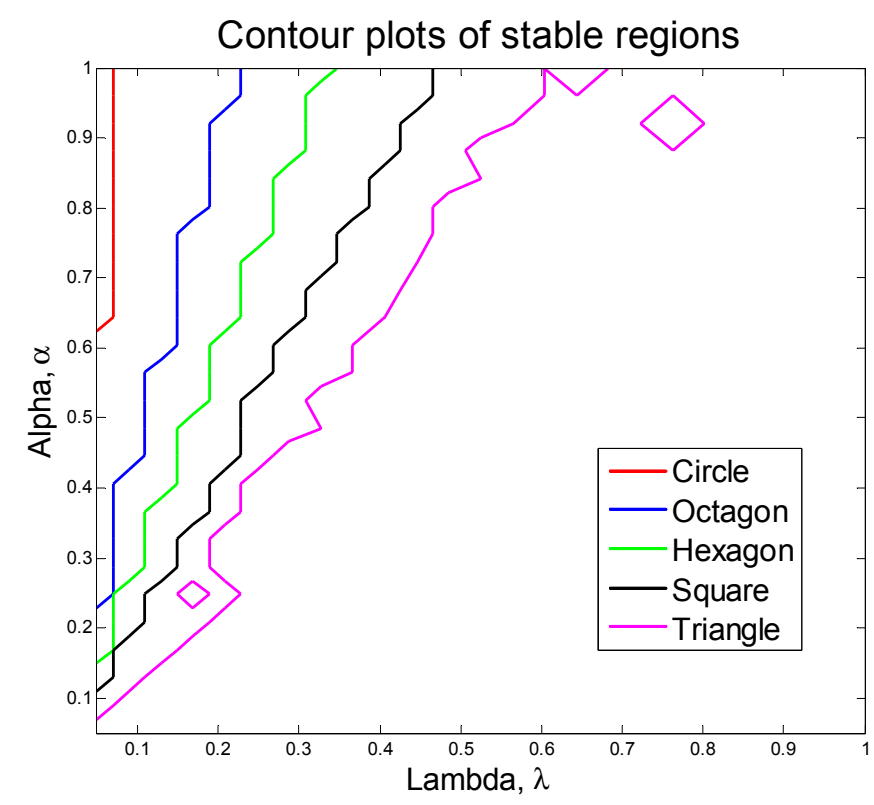

Figure 5 Contour plot displaying the regions of stability for different shapes under the active contour model. The region above the contour lines is undesirable as it corresponds to parameter values in category A. 
Chopin, Miklavcic, Laga. Mathematical and Computational Modelling for the Phenotypic Analysis of Plants

\section{ACKNOWLEDGEMENTS}

This project is supported with funding from the Australian Centre for Plant Functional Genomics and a grant from the South Australian State Government's Premiers Science and Research Fund.

\section{REFERENCES}

Allili M. S. and Ziou, D. (2008). An approach for dynamic combination of region and boundary information in segmentation, Int. Conf. on Pattern Recognition, 1-4.

Argialas, D. and Karantzalos, K. (2009). A region-based level set segmentation for automatic detection of man-made objects from aerial and satellite images, Photogrammetric Eng. and Remote Sensing, 667-677.

Berg, J. (1998). Estimation of parameters appearing in the level set evolution equation, Proc. of the $37^{\text {th }}$ IEEE Conf. on Decision and Control, 2323-2328.

Cai, X., Sowmya, A. and Trinder, J. (2006). Learning parameter tuning for object extraction, Proc. of the $7^{\text {th }}$ Asian Conf. on Comp. Vision, 1, 868-877.

Cai, X. and Sowmya, A. (2009). Learning to tune level set methods, Int. Conf. Image and Vision Computing New Zealand, 310-315.

Farag, A. A., Hassouna, M.A. and Abdel-Hakim, A.E. (2007). PDE-based robust robotic navigation, Image and Vision Computing, 27, 10-18.

Frick, R., Miklavcic, S., Golzarianm M., and Cai, J. (2011). Segmentation of cereal plant images using level set methods - a comparative study, Int. Journal of Information and Electronics Engineering, 1, 72-78.

Iakovidis, D. K., Savelonas, M.A., Karkanis, S.A. and Maroulis, E. (2007). A genetically optimized level set approach to segmentation of thyroid ultrasound images, Int. Journal of Artificial Intelligence, Neural Networks and Complex Problem-Solving Technologies, 193-203.

Kass, M. and Witkin, A. (1988). Snakes: Active contour models, Int. Journal of Computer Vision, 1, 321331.

Keuper, M., Schmidt, T., Padeken, J., Heun, P., Palme, K., Burkhardt, H. and Ronneberger, O. (2010). 3D deformable surfaces with locally self-adjusting parameters - A robust method to determine cell nucleus shapes, Int. Conf. on Pattenr Recognition, 2254-2257.

Kussener, F. (2011). Active contour: a parallel genetic algorithm approach, Int. Conf. on Swarm Intelligence, $1-9$.

Museth, K., Osher, S., Sapiro, G., Whitaker, R., Breen, D. and Fedkiw, R. (2004). Level set and PDE methods for computer graphics, ACM SIGGRAPH 2004 course notes.

Mylona, E. A., Savelonas, M.A. and Maroulis, D. (2012). Entropy-based spatially-varying adjustment of active contour parameters, Int. Conf. on Image Processing, 2565-2568.

Ring, W. and Hintermuller, M. (2004). A level set approach for the solution of a state-constrained optimal control problem, Numerische Mathematik, 98, 135-166.

Rivollier, S., Debayle, J. and Pinoli, J. C. (2011). Shape diagrams for 2D compact convex sets - Part 1: Analytic convex sets, Australian Journal of Mathematical Analysis and applications, 1-27.

Stijnen, B., De Dobbelaer, B., Vandermeulen, D., Suetens, P., Smeets, D. and Loeckx, D. (2009). Semiautomatic level set segmentation of liver tumors combining a spiral-scanning technique with supervised fuzzy pixel classification, Medical Image Analysis, 13-20.

Tian, Y., Duan, F., Zhou, M. and Wu, Z. (2013). Active contour model combining region and edge information, Machine Vision and Applications, 24, 47-61. Zhu, Y., Cheng, S. and Goel, A. (2011). A nonparametric segmentation method based on structural information using level sets, Proc. SPIE 7962, Medical Imaging 2011: Image Processing, 79623C-1 - 79623C-6. 